\title{
A Cost Function for QoS-Aware Routing in Multi-tier Wireless Multimedia Sensor Networks
}

\author{
Stéphane Lohier $^{1}$, Abderrezak Rachedi $^{1}$, and Yacine Ghamri-Doudane ${ }^{2}$ \\ ${ }^{1}$ IGM - Université de Paris-Est Marne-la-Vallée, 75420 Champs sur Marne, France \\ ${ }^{2}$ ENSIIE, 1Square de la résistance, 91025 Evry Cedex, France \\ lohier@univ-mlv.fr, rachedi@univ-mlv.fr, ghamri@ensiie.fr
}

\begin{abstract}
Wireless Multimedia Sensor Networks (WMSNs) are composed of small devices that are able to capture video or audio information and to transmit it over wireless channels. The development of wireless technologies, such as: WiFi, Bluetooth, Zigbee, and UWB, encourages the emergence of heterogeneous networks. However, only a few existing solutions take into account the constraints of multi-tier and multi-MAC wireless sensor networks. In this paper, we propose a cost function coupled to a new generic (i.e. independent from any MAC protocol) cross-layer routing protocol adapted to multimedia traffic over hierarchical and heterogeneous networks. The goal of our proposed protocol is to dynamically assess the routing process cost and the requirement of the multimedia application in order to ensure a sufficient quality of service (Soft QoS). Furthermore, the cross-layer approach is needed to use the physical and MAC parameters on the routing operation. Simulation results show that our solution is efficient and gives better results than classical protocols.
\end{abstract}

Keywords: WMSNs, heterogeneous network, cost function, cross-layer, QoS.

\section{Introduction}

The popularity of Wireless Sensor Networks (WSNs) is growing with the technological advancement that enables to design a small and smart device able to capture the multimedia information such as CMOS cameras and microphones [1]. This growing interest enables the development of particular wireless sensor networks called Wireless Multimedia Sensor Networks (WMSNs). Furthermore, WMSNs enable to enlarge WSNs application field: it can be used for multimedia surveillance systems against crime and terrorist attacks, car traffic monitoring in big cities or highways, environmental monitoring through acoustic and video data, etc.

Unlike classical WSNs, WMSNs have specific characteristics such as: 1) high bandwidth demand due to multimedia content, 2) specific QoS requirements that are dependent on the application, and 3) important power resource consumption due to high volumes of data to transmit (or forward) by battery-constrained devices. One should also note that due to the nature of WMSN applications, the node's mobility is not high. Obviously, WMSNs are more challenging than classical WSNs since additional constraints and parameters are introduced. That is why Akyildiz et al. [2] proposed and advocated for a multi-tier architecture. This architecture ensures the network 
scalability and enables the use of heterogeneous elements. These architectures provide an efficient cost-performance tradeoff while taking into account less expensive and resource-constrained scalar sensors and high power superior elements such as multimedia sensors. In this architecture, the network is divided into clusters. Each cluster elects a cluster head that has sufficient resources.

In this paper, we use the multi-tier architecture to ensure the scalability and the soft-based quality of services [2]. We focus on the routing process to increase the throughput and reduce the packet loss and the delay. Therefore, in order to optimize the resources and make the multimedia traffic routing more efficient, we propose a new routing protocol based on a cost-function called HQAX (Hierarchical QoS Aware Cross-layer routing protocol). The basic idea of this cost function is to take into account not only the routing parameters, but also the physical and MAC layers parameters such as the channel quality, the SNR, and the number of ACK failures. Our design is thus a cross-layer design enabling to take into account the physical and MAC layers parameters at the routing level. We show that with such a mechanism, we can improve the network throughput; reduce the packet loss and the delay in indoor environments. The impact of the environment on the communication channel quality is very significant. For instance, in the case of an outdoor environment, called free-space, the channel quality is better than when many obstacles are present inside a building. However, most applications of WMSNs, like building surveillance, are in an indoor environment. That is why we focus on such realistic scenarios to validate our proposed protocol.

After analyzing and studying WMSNs requirements and their potential implementation using multi-tier architecture, our contributions can be summarized in the three following points:

- Proposition of a new routing protocol (HQAX) based on a cost function in order to improve the throughput and to reduce the delay and packet losses.

- Definition of a cost function in order to optimize the resources and to make the multimedia traffic routing more efficient (the cross-layer approach is used for the cost function in order to take into account the physical and MAC layers parameters at the routing level).

- Implementation and evaluation of the proposed routing protocol in comparison with other existing protocols like AODV and OLSR which had been adapted to cope with the features and characteristics of multi-tier WMSNs.

The rest of the paper is organized as follows. In Section 2, we present the related works. In Section 3, we present and detail our proposed routing protocol. A description of our cost function follows in Section 4. In Section 5, we evaluate and compare our solution with existing routing protocols. Section 6 concludes the paper.

\section{Related Works}

Many routing protocols are proposed in literature. In proactive networks, Heinzelman et al. proposed a Low-Energy Adaptive Clustering Hierarchy (LEACH) for wireless sensor networks [3]. LEACH is a dynamic clustering protocol designed with energy constraint consideration. Lindsey et al. proposed an improvement of LEACH called Power-Efficient Gathering in Sensor Information Systems (PEGASIS) [4]. The basic 
idea of PEGASIS is to form a chain among the sensor nodes where each node will receive from and transmit to a close neighbor in order to reduce the energy consumption. These protocols focus on the dynamic clustering issue. Once the clusters are established and the cluster head role assigned, it is still necessary to ensure the quality of services required by multimedia traffic. Our objective is to target this second problem.

In the QoS-aware routing protocols class, we can quote some proposals for WSNs. One of them is the Sequential Assignment Routing (SAR) [5]. SAR creates trees routed from a one-hop neighbor of the sink by taking into account the QoS metric, the energy resource on each path and the priority level of each packet. However, the SAR protocol does not focus on the throughput maximization. Some other routing protocols also integrate the energy parameters in addition to other QoS parameters. An example of these is the SPEED protocol [6]. SPEED is a real-time communication protocol for sensor networks. The protocol provides three types of real-time communication services, namely real-time unicast, real-time area-multicast and real-time area-anycast. However, SPEED needs localization algorithms to achieve high scalability and avoid a flooding operation to discover new paths. Another protocol, proposed by C.G. Lee $e t a l$. and called Multipath Multi-SPEED Protocol (MMSPEED) [7], adopts a probabilistic approach to offer QoS assurance in wireless sensor networks. To do so, it uses a cross-layer approach between the network and the MAC layers. However, MMSPEED assumes that each node is equipped with a GPS chip which may not be a suitable assumption in WSNs, especially in indoor environments.

An energy-aware routing protocol in a cluster-based architecture is presented in [8]. It uses a cost function based on energy saving that is coupled with a source routing protocol. Unlike our approach, the topology is multi-gateway and non multi-tier (multi-MAC). Furthermore, the protocol is specific to the use of a TDMA MAC layer.

\section{Hierarchical QoS Aware Cross-Layer Routing Protocol}

\subsection{Context}

Figure 1 describes our proposal for a multi-tier architecture inspired by the work of I.F. Akyldiz et al. [2]. In this heterogeneous and hierarchical architecture, each tier corresponds to a category of video sensors with increased capabilities in terms of camera resolution, processing, storage and transmission. For the third tier, the sensors can be CMUCam (weak resolution of 160x255) coupled with microcontrollers allowing a minimum processing and not very greedy transmissions like in ZigBee, Bluetooth or UWB standards. The second tier can be made up of a webcam and microcontrollers with more processing, more storage and mixed transmissions, ZigBee and 802.11 for instance. The first tier is connected to the sink (multimedia server) and includes high resolution cameras coupled with laptops.

For each tier, our proposal is to organize the topology into clusters with a Cluster Head $(\mathrm{CH})$, Cluster Routers (CR) allowing multi-hop routing when necessary, and Cluster Terminals (CT), only able to capture video information and to transmit it. In order to limit the interferences, the nearby clusters can use distinct transmission channels. The sensors at the various levels can be moved but are not permanently mobile.

Moreover, the processing essentially carried out in the $\mathrm{CHs}$ and towards the sink, includes specific operations like compression, data aggregation (images from different 


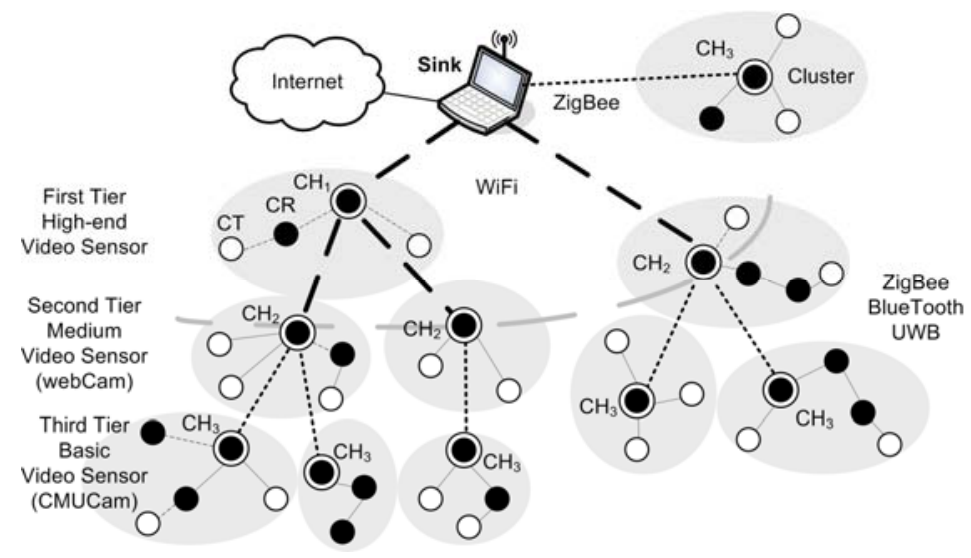

Fig. 1. Multi-tier Architecture of WMSN

scenes in the same flow) and data suppression (redundant images from various sensors). These different characteristics of the multi-tier WMSN architecture (clustered architecture, many-to-one flows, heterogeneous capabilities, and processing into the $\mathrm{CHs}$ ) make us believe that a hierarchical routing is the most suited one.

\subsection{The Goals of HQAX}

The goal is not to propose an additional new routing protocol but rather to adapt existing solutions in order to have a QoS-aware routing protocol:

- linked to the application : the sensor networks are application-aware;

- based on a cross-layer cost function with routing, MAC and PHY parameters related to the multimedia feature of the flows;

- adapted to a hierarchical and multi-tier (multi-MAC) architecture;

- for many-to-one transmissions, and not for many-to-many like in current ad hoc network protocols like AODV or OLSR;

- generic, i.e. non related to a specific MAC layer but nevertheless compatible with the existing sensor routing protocols (ZigBee);

- with a limited overhead thanks to the combination of the cluster association and the route setup processes;

- easy to implement in the different devices of a real test bed.

The following sections describe our QoS-aware hierarchical routing inside clusters, whatever the tier is, and between the clusters of the various tiers. The QoS route setup is the first step of our solution. Indeed, the network organization must remain evolutionary according to the periodic requests from nodes to join or leave a cluster and to the needs of the sink-application which will select, starting from descriptors (fixed image, possible resolution...), the transmitting sensors as well as the characteristics of the transmitted flows (zone, resolution, compression ratio, cropping...). Thus, the objective here is not to constantly guarantee a QoS but to choose and receive pictures of a sufficient quality (soft QoS). The idea is thus to use the best available routes. 


\subsection{Intra-cluster QoS Routing}

The QoS routing proposed in each cluster is proactive and includes 6 stages for the cluster self-organization and the route setup procedures (Figure 2). As indicated previously, we drew our inspiration from the existing solutions like ZigBee for the association process which is adapted to cope with the multi-tier feature of the WMSNs as well as with the QoS requirements of transmitted flows.

1. Each node self-determines its potential role $(\mathrm{CH}, \mathrm{CR}$ or $\mathrm{CT})$ in a cluster according to fixed or periodically re-evaluated criteria:

- sufficient storage and energy (in comparison to specific thresholds for each role);

- for $\mathrm{CH}$ : transmission capacity (presence of other devices corresponding to the tier) and computational capacity for aggregation, suppression, compression....

2. Each $\mathrm{CH}$ initiates a cluster (scan channels, select a channel, select a cluster id...).

3. CR and CT look for a cluster (Figure 3):

- CR/CT broadcast a discovery message: Cluster_Discovery_Request (Scan Channels...);

- response of the nearby CHs (and/or CR in the case of a multi-hop distance) with a Cluster_Discovery_Response (Cluster Description...).

4. Evaluation of a $\operatorname{cost}^{1} C_{i j}$ for the links involved. This evaluation starts at stage 3 with the exchange of the Cluster_Discovery.

5. $\mathrm{CR}$ and $\mathrm{CT}$ choose a $\mathrm{CH}$ (or a $\mathrm{CR}$ ) according to the previous QoS evaluation and join a cluster:

- CR/CT send a message Cluster_Join_Request (Cluster id...);

- response of the selected CH (or CR) with a Cluster_Join_Response (Cluster id, Network addresses...).

The associations of the CRs and the CTs are carried out in a recursive way: for the multi-hop routing, a CT out of the $\mathrm{CH}$ range has to wait for the association of a nearby CR to obtain an answer and thus join the cluster.

6. The $\mathrm{CR}$ informs its $\mathrm{CH}$ (or its nearby $\mathrm{CR}$ which is closer to the $\mathrm{CH}$ ) about its router's role:

— the CR sends a message Cluster_Router_Request (Cluster id...);

- response of the CH (or CR) with a Cluster_Router_Response (Cluster id, Network address block...). In its response, the $\mathrm{CH}$ specifies the address block (or a sub-block in the case of a $\mathrm{CR}$ answering) which can be used by the requesting $\mathrm{CR}$ for its $\mathrm{CT}$ and its lower level CR.

After one or several exchange cycles, each $\mathrm{CR}$ or $\mathrm{CT}$ knows the address of its $\mathrm{CH}$ or its nearby $\mathrm{CR}$ (the one with the lowest cost towards the $\mathrm{CH}$ ). Similarly, each $\mathrm{CH}$ or $\mathrm{CR}$ has a routing table for all its nodes. The routing is hierarchical: all data pass through the $\mathrm{CH}$. According to the network dynamics, the various stages are periodically re-launched.

\footnotetext{
${ }^{1}$ The cost function integrating the QoS parameters is described in Section 4.
} 


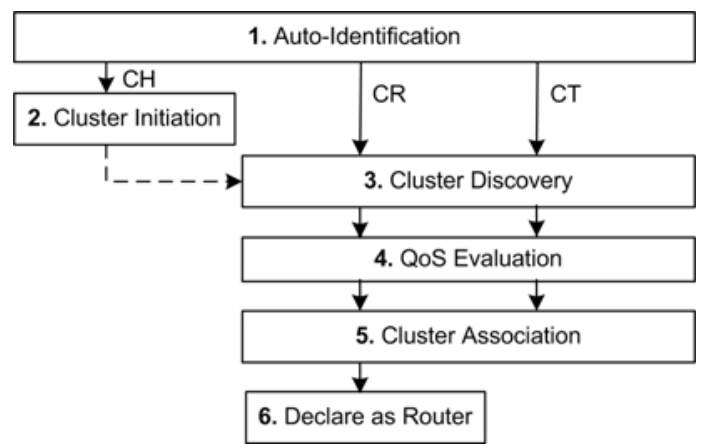

Fig. 2. Intra-cluster Routing Algorithm

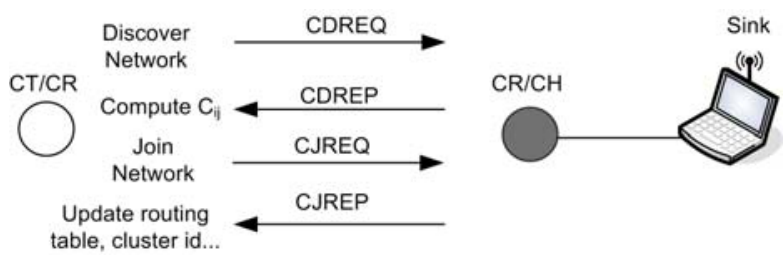

Fig. 3. Intra-cluster Association Process

\subsection{Inter-tier QoS Routing}

The routing between the CHs of various tiers (Figure 2) proceeds in 5 stages according to the same hierarchical and recursive principle:

1. The $\mathrm{CH}_{3}\left(\mathrm{CH}\right.$ of third tier) broadcast a request to know the $\mathrm{CH}_{2}$.

2. The $\mathrm{CH}_{3}$ choose a $\mathrm{CH}_{2}$ according to a cost estimated on the links.

3. The $\mathrm{CH}_{2}$ broadcast a request in their turn on the corresponding interface to know the $\mathrm{CH}_{1}$.

4. The $\mathrm{CH}_{2}$ choose a $\mathrm{CH}_{1}$ according to a cost estimated on the links.

5. The $\mathrm{CH}_{1}$ broadcast a request to know the sink. In this last case, the cost is also computed to evaluate if, according to its location and environment, the $\mathrm{CH}_{1}$ can get a sufficient QoS on the link towards the sink.

After sufficient exchanges, each $\mathrm{CH}_{n}$ knows the address of its $\mathrm{CH}_{n-1}$ and the sink (transmitted with the response to the broadcast). Each node in each cluster can thus transmit towards the sink and vice-versa (the sink knows the $\mathrm{CH}_{1}$ which knows the $\left.\mathrm{CH}_{2} \ldots\right)$.

\section{Utility Theory-Based Cost function}

During the messages exchange of the discovery stage (between $\mathrm{CT} / \mathrm{CR}$ and $\mathrm{CR} / \mathrm{CH}$ or between $\mathrm{CH}_{\mathrm{i}}$ and $\mathrm{CH}_{\mathrm{i}+1}$ ), the cost on each possible link is periodically estimated and compared (i) to a threshold to decide if it meets the conditions (noise, contentions...) 
to get a sufficient QoS and (ii) with the costs on the other links to choose the most efficient $\mathrm{CR} / \mathrm{CH}$. The cost function on a link between two nodes $i$ and $j$ ( $j$ being the closest to the sink) can be expressed according to the chosen QoS metrics as follows:

$$
C_{i j}=\sum_{k} c_{k} \times f_{k}\left(x_{i j}^{k}\right)
$$

where $x_{i j}^{k}$ is the value of metric $k$ relatively to the link between two nodes $i$ and $j, c_{k}$ is the preference weight of metric $k\left(\right.$ where $\left.\sum_{k} c_{k}=1\right)$, and $f_{k}(:)$ is a normalized function. In our case, we suggest choosing the five following metrics:

$$
C_{i j}=c_{1} \times f_{1}\left(\text { delay }_{i j}\right)+c_{2} \times f_{2}\left(S N R_{j i}\right)+c_{3} \times f_{3}\left(e_{i j}\right)+c_{4} \times f_{4}\left(\text { energy }_{i}\right)+c_{5} \times f_{5}\left(\text { energy }_{j}\right)
$$

$-f_{l}\left(\right.$ dela $\left._{i j}\right)$ is a function of the delay for a data packet transmitted from $i$ to $j$;

$-f_{2}\left(S N R_{j i}\right)$ is a function of the Signal/Noise Ratio assessed from $j$ to $i$;

$-f_{3}\left(e_{i j}\right)$ gives the error rate for the data packets transmitted from $i$ to $j$;

$-f_{4}\left(\right.$ energy $\left._{i}\right)$ and $f_{5}\left(\right.$ energy $\left._{j}\right)$ give the remaining energy in $i$ and $j$ respectively.

The choice of weights $c_{1}$ to $c_{5}$ depends on the application and on the type of traffic (for instance, for streaming applications the delay is more important than the loss rate).

Besides, for "real time" applications, it is necessary to control the end-to-end delay. Rather than using higher level protocols like RTP and RTCP which involve an overhead, we can evaluate this global delay at the routing level, starting from the delay on each link and the knowledge of the route towards the sink, both data being provided by our routing protocol. The loss rate on a path from a CT towards the sink can also be evaluated, starting from the successive loss rates and the knowledge of the route.

The remaining question is related to the form to be used for the normalized $f_{k}(:)$ functions. To answer this question, the most suitable form can be found in the wellknown concept of utility functions. Indeed, the utility theory correctly models the soft tuning of the perceived quality in modern networks.

The utility theory is used for multi-criteria selection which is a classical problem in economics and in many other fields. It is used to give a measure of the relative satisfaction from (or desirability of) the consumption of various goods and services. The use of the utility theory has been extended to many other fields where it is used as a scoring method that quantifies the score (suitability level, value, worth) of a particular choice compared to another one. In our current work, we suggest to use it to quantify the utility of using a link in a QoS-aware routing process. The utility of the link corresponds here to the cost function on the link between both nodes $\mathrm{i}$ and $\mathrm{j}$. The utility theory is thus used as the basis of our cost function. The normalized function is introduced to express different characteristics of different units with a comparable numerical representation. Different normalized functions were used in literature to solve several QoS issues in communication networks. Among them, we can quote: the linear piecewise form [9], the logarithm form [10], the exponential form [10], and sigmoid forms $[12,13]$. Among these, the most commonly used normalized functions are the sigmoidal (S-shaped) functions. Indeed, sigmoidal functions are well-known functions often used to describe QoS perception [13]. Thus we chose here to use these functions. More precisely, we consider the following analytic expression for the sigmoid form: 


$$
f(x)=\frac{\left(x-x_{m}\right)^{\zeta}}{1+\left(x-x_{m}\right)^{\zeta}}
$$

where $x_{m}>0$ and $\zeta \geq 2$ are tunable parameters, that differentiate the users' utilities. It is also assumed that the utilities are normalized to their highest limit, i.e. the asymptotic value of $f(x)$ for large $x$ is considered to be equal to 1 . This is only done for the sake of simplicity [13]. In other more complicated scenarios, different maximum utilities can also be considered.

\section{Performance Evaluation}

\subsection{Context}

To analyze the improvements registered by the HQAX protocol and the cost function, we implemented a set of simulations using the NS-2 simulator (version 2.33). The selected scenario implements a hierarchical multi-tier topology. Insofar as NS-2 is not conceived to bring together various interfaces and various MAC layers in the same node or in the same wireless network, only the 802.11 MAC layer will be used in the clusters of the different tiers. The objective being here to test the efficiency of the cost function integrated into our routing protocol, in comparison with current routing protocols, the simulations can be implemented without any real multi-MAC topology.

We chose to use the "shadowing" radio-propagation model, which is more realistic as it takes into account the shadowing and the fading effect that are common in indoor and outdoor environments with potential mobile obstacles. Moreover, to highlight the interest of the cost function, we chose the appropriate parameters for an indoor environment corresponding for example to a building surveillance application (see Table 1).

Table 1. Simulation Parameters

\begin{tabular}{ll}
\hline Nodes number & 31 \\
Simulation Area & $135 \times 84$ meters \\
Simulation Time & 20 seconds \\
Traffic Type, Packet size, Period & CBR, 1000 Bytes, 1.5 to $50 \mathrm{~ms}$ \\
Radio-propagation model & Shadowing \\
Radio-propagation parameters & Reference distance $=10 \mathrm{~m}, \beta=5, \sigma=7 \mathrm{~dB}$ \\
Transmission Range & $25-50$ meters \\
\hline
\end{tabular}

The simulated network includes 30 nodes and a sink distributed on a $135 \times 84 \mathrm{~m}$ area (see Figure 4). The role of each node ( $\mathrm{CH}, \mathrm{CR}$ or $\mathrm{CT}$ ), which should depend on the available resources, is fixed at the beginning. The distances between the nodes are such that a $\mathrm{CT}_{\mathrm{n}}$ or a $\mathrm{CR}_{\mathrm{n}}$ has always the choice between at least two potential routes to join its $\mathrm{CH}_{\mathrm{n}}$. It is the same for a $\mathrm{CH}_{n}$, during the association with its $\mathrm{CH}_{\mathrm{n}+1}$. Let us note that $\mathrm{CH}_{2}$ or $\mathrm{CH}_{3}$ can directly join the sink as this latter is assumed to have all MAC devices according to the use of multi-tier architecture. In addition, although the 802.11 MAC layer is common to all nodes, a CT (or a CR) can only join a CR or a $\mathrm{CH}$ at the same level. In this topology, all nodes can be multimedia sensors so all 
nodes are modeled as CBR sources. We tested several scenarios of sources activation with a number of CBR sources ranging between 1 and 30 (in this last case, all nodes are sources). The total duration of each simulation is 20s (sufficient in all cases to get a stable network) and the CBR sources are activated every $0.1 \mathrm{~s}$ to avoid synchronized broadcast (in a multimedia sensor network, all cameras are seldom activated at the same time). In addition, in this type of applications (monitoring, surveillance ...), the nodes are often fixed or very slightly mobile. We thus chose a null mobility during the simulation.

The cost function implemented for the simulation is adapted to 802.11 devices and takes into account four parameters:

$$
C_{i j}=c_{1} \times f\left(\text { delay }_{i j}\right)+c_{2} \times f\left(\operatorname{Pr}_{i j}\right)+c_{3} \times f(\text { hops })+c_{4} \times f\left(P L R_{i j}\right)
$$

- The delay on the link between nodes $\mathrm{i}$ and $\mathrm{j}$ is simply evaluated using a timestamp transmitted to the CDREP association packet.

- The received power $P r$ is evaluated when receiving the CDREP packet. The number of hops is measured from the source to the sink.

- The Packet Loss Rate (PLR) is calculated on the node which transmits the CDREP packet and then transmitted in this same packet to the node having requested the association or to the node relaying the answer. The PLR is calculated from the MAC-MIB 802.11 information and then uploaded at the routing level:

$$
P L R_{M P D U}=\frac{\text { ACKFailureCount }}{\text { TransmittedFragmentCount }+ \text { ACKFailureCount }}
$$

Coefficients $c_{1}$ to $c_{4}$ are optimized to get the best results in the selected context. To evaluate the HQAX performances and the associated cost function, we chose to compare it with the AODV and OLSR protocols which we adapted to a multi-tier architecture.

Concerning AODV, several reasons justify this choice:

- it is a standardized reference protocol for ad hoc networks and it is largely used in sensor networks: AODV is included in ZigBee [14];

- it is a generic protocol and it is not associated to a specific application or a particular access method as opposed to LEACH (based on dynamic clustering, energy saving and TDMA distribution) or SPEED (real time with geo-localization assumptions);

- AODV is implemented in the most common simulators like NS-2, as opposed to some specific WSN protocols.

In addition, the choice of OLSR (the implementation used here for NS-2 is UMOLSR [15]) enables to compare HQAX to a proactive reference protocol in which the relay nodes (MPR) are elected according to the number of neighbors. For these simulations, AODV and OLSR were adapted, including cross-layer interactions with the MAC layer, to respect the multi-tier architecture (AODV-mt and OLSR-mt on the following figures). These adaptations are made so that: a $\mathrm{CT}_{\mathrm{n}} / \mathrm{CR}_{\mathrm{n}}$ can only join a $\mathrm{CR}_{\mathrm{n}}$ or a $\mathrm{CH}_{n}$; a $\mathrm{CH}_{\mathrm{n}}$ can directly join the sink or must pass by $\mathrm{CH}_{\mathrm{n}+1}$; a CT does not relay a route request broadcast; a CT cannot be elected as an MPR (in the case of OLSR). 


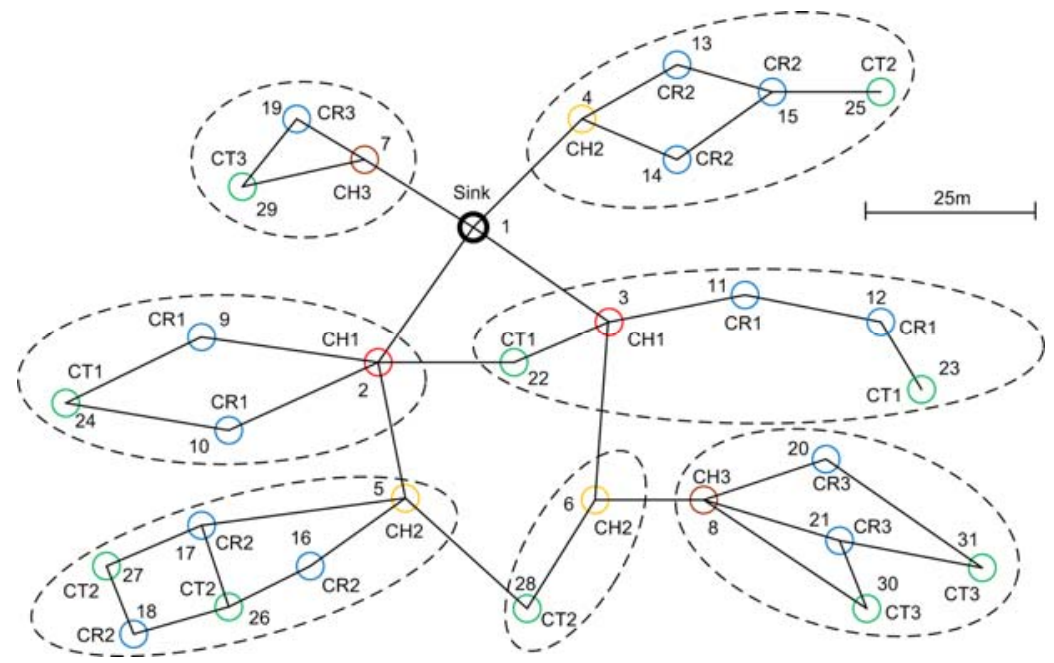

Fig. 4. Multi-tier hierarchical scenario

\subsection{Results}

Figure 5 presents the evolution of the average end-to-end delay for the CBR packets on all routes towards the sink, when the number of sources varies from 1 to 30 . The results are slightly better with OLSR but with a much higher loss rate (Figure 7): the delays on the intermediate links between $\mathrm{CR}$ and $\mathrm{CH}$ can be shorter because many packets are lost on the way. For AODV, the average delay fluctuates between 3 and 8 ms showing a great dependence on the number of sources and the geographical distribution of nodes. Finally, HQAX presents a stable average delay, whatever the number of active sources, and relatively low if we take into account the number of packets actually transmitted. This shows the efficiency of the cost function which integrates the delay on the links.

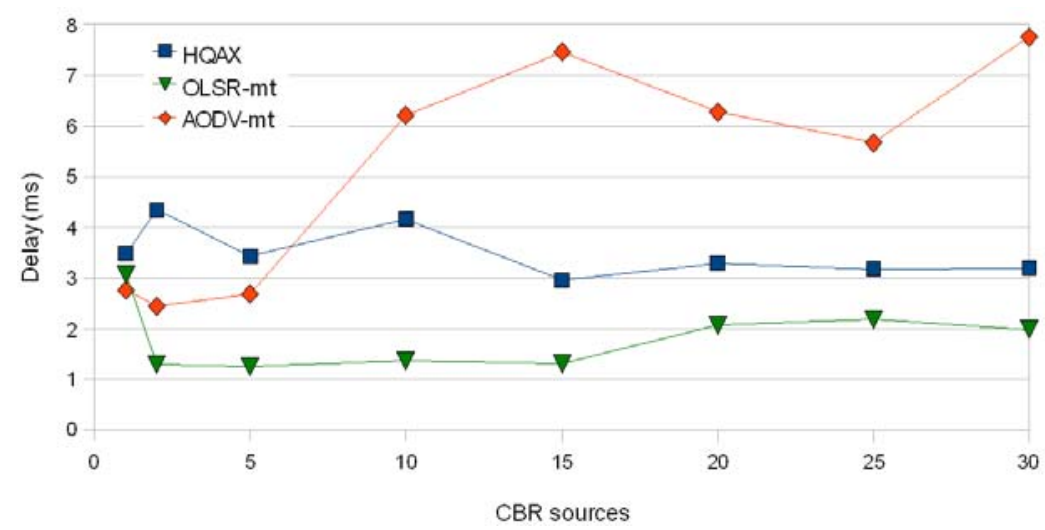

Fig. 5. Average Packet Delay / Number of CBR sources 
Figure 6 shows the evolution of the average global throughput for all active CBR sources. The results are very close for a low number of sources and are slightly better for HQAX for more than 5 active sources. The evaluation in the cost function of the received power and of the loss rate during the association process makes it possible to reduce the risk of contentions on the selected links.

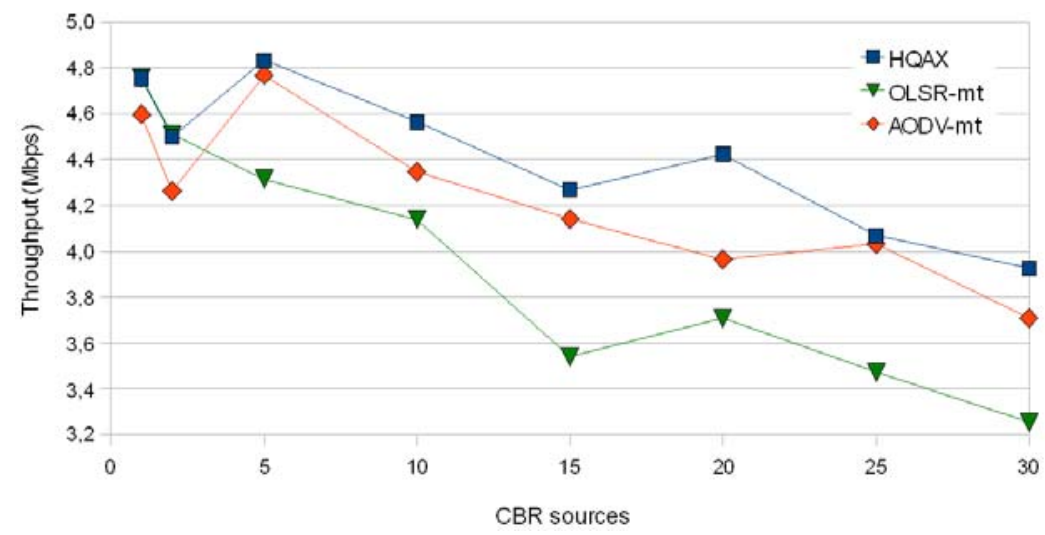

Fig. 6. Average Global Throughput / Number of CBR sources

In Figure 7, the packet loss rate for all active routes increases very quickly with OLSR, mainly because of a bad load balancing on the MPR which are elected according to the number of neighbors and not to the hierarchical organization of the network. AODV enables, with its error packet transmission mechanism, to mitigate the loss duration but at the expense of the overhead (see Figure 8). Whatever the number of sources, the loss rate remains lower than $1 \%$ with HQAX. This confirms the interest to upload at the routing level the number of non-received acknowledgements which is computed at the MAC level.

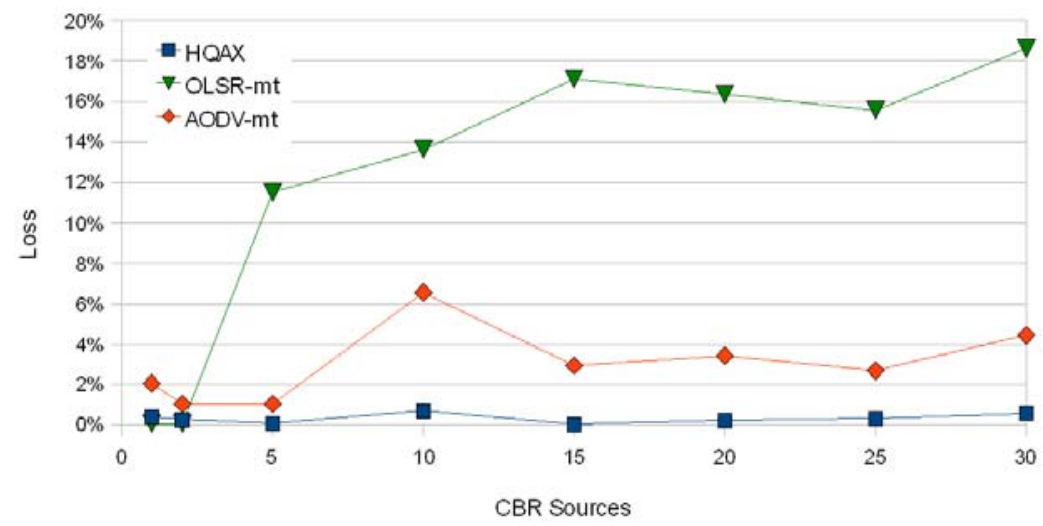

Fig. 7. Packet Loss / Number of CBR sources 
The relative overhead of control packets (Figure 8) remains lower than 5\% with OLSR and HQAX. Let us remind that for OLSR, the frequencies of Hello and TC messages are optimized for a not very dense and not mobile context. This overhead is much more important with AODV starting from 10 active sources. Although AODV is modified for our multi-tier architecture, the unsolved contentions can be numerous and the error messages can lead to broadcast new requests, creating a strong overhead.

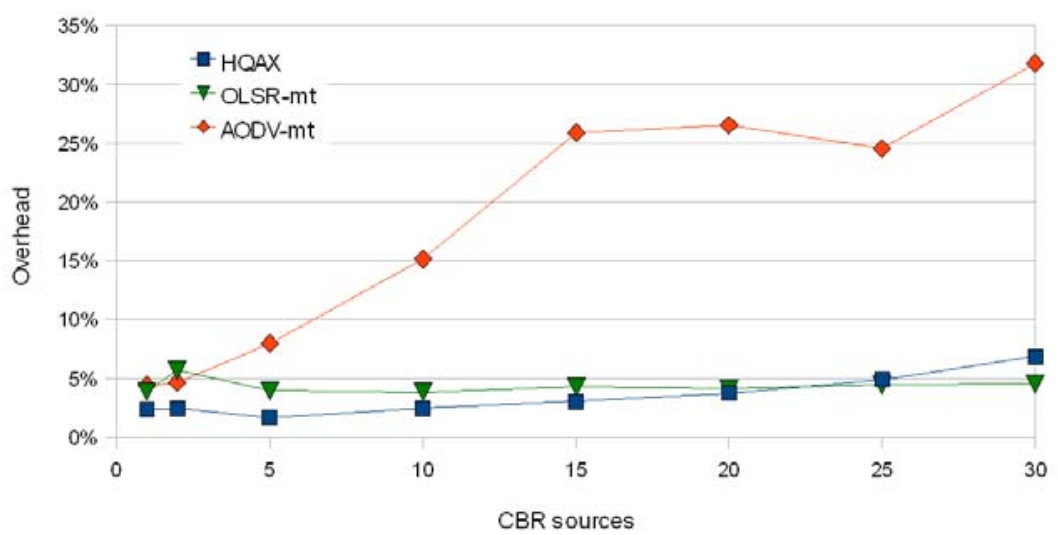

Fig. 8. Control Packet Overhead / Number of CBR sources

\section{Conclusion and Perspectives}

In this paper, we presented a solution including a reactive routing protocol associated with a cost function adapted to a heterogeneous and hierarchical WMSN architecture divided into clusters. This solution enables the sink to collect multimedia flows from different kinds of sensors, in the various tiers, with a sufficient quality of service. The advantages of this solution are on one hand its generic feature: it does not depend on a specific MAC layer or a particular access method, and on the other hand, the fact that it is compatible with the existing link and routing layers used by wireless sensor networks: the MAC parameters used in the cost function are generic and exist in both 802.11 and 802.15.4 MIBs; furthermore, the reactive routing approach used in HQAX is compatible with that used in ZigBee.

Our simulation study shows the efficiency of this solution, particularly in terms of throughput, loss rate, and control packet overhead. This first evaluation campaign will have to be confirmed by real test-bed experiments. Such a test-bed should integrate various devices corresponding to the different WMSN tiers (WiFi and ZigBee enabled sensors). The generic and compatibility features of our proposal should facilitate such an implementation. This experimental evaluation constitutes the target of our future work. 


\section{References}

1. James, D., Klibanov, L., Tompkins, G., Dixon-Warren, S.J.: Inside CMOS Image Sensor Technology, Chipworks White Paper,

http: //www. chipworks.com/resources/whitepapers / Inside-CMOS.pdf

2. Akyildiz, I.F., Melodia, T., Chowdhury, K.R.: A survey on wireless multimedia sensor networks. Journal of Computer Networks 51(4) (2007)

3. Heinzelman, W., Chandrakasan, A., Balakrishnan, H.: Energy- Efficient Communication Protocol for Wireless Microsensor Networks. In: Proceedings of the Hawaii International Conference on System Sciences, January 4-7. IEEE Press, Los Alamitos (2000)

4. Lindsey, S., Raghavendra, C.S.: PEGASIS: Power-Efficient GAthering in Sensor Information Systems. In: IEEE Aerospace Conference Proceedings, pp. 1125-1130 (2002)

5. Sohrabi, K., et al.: Protocols for self-organization of a wireless sensor network. IEEE Personal Communications 7(5), 16-27 (2000)

6. He, T., Stankovic, J.A., Lu, C., Abdelzaher, T.: SPEED: A stateless protocol for real-time communication in sensor networks. In: The Proceedings of International Conference on Distributed Computing Systems, Providence, RI (May 2003)

7. Lee, C.-G., Ekici, E., Felemban, E.: MMSPEED: Multipath Multi-SPEED Protocol for QoS Guarantee of Reliability and Timeliness in Wireless Sensor Networks Full text. IEEE Transactions on Mobile Computing 5(6), 738-754 (2006)

8. Younis, M., Youssef, M., Arisha, K.: Energy-Aware Routing in Cluster-Based Sensor Networks. In: The Proceedings of the 10th IEEE/ACM International Symposium on Modeling, Analysis and Simulation of Computer and Telecommunication Systems (MASCOTS2002), FortWorth, TX (October 2002)

9. Ormond, O., Muntean, G., Murphy, J.: Economic model for cost effective network selection strategy in service oriented heterogeneous wireless network environment. In: Proc. of NOMS 2006, Canada (2006)

10. Ormond, O., Muntean, G., Murphy, J.: Network selection decision in wireless heterogeneous networks. In: Proc. of IEEE 16th Intl Symposium on Personal, Indoor and Mobile Radio Communications, Berlin (2005)

11. Wang, H.J., Katz, R.H., Giese, J.: Policy-enabled handoffs across heterogeneous wireless networks. In: IEEE Workshop on Mobile Computing Systems and Applications (1999)

12. Pal, S., Das, S., Chatterjee, M.: User-Satisfaction based Differentiated Services for Wireless Data Networks. In: Proc. of IEEE International Conference on Communications (ICC), vol. 2, pp. 1174-1178 (2005)

13. Badia, L., Lindstrom, M., Zander, J., Zorzi, M.: An economic model for the radio resource management in multimedia wireless systems. Computer Communications 27(11), 10561064 (2004)

14. ZigBee Specifications, ZigBee Alliance (December 2006), http : / /www . zigbee . org

15. Implementation of OLSR for NS,University of Murcia (Spain), http: //masimum.dif.um.es/?Software:UM-OLSR 